\title{
Flight Readiness of Mochii S: Portable Spectroscopic Scanning Electron Microscope Facility on the International Space Station (ISS)
}

James E. Martinez ${ }^{*}$, Christopher S. Own², Matthew P. Galeano ${ }^{1}$, Gerward Weppelman², Donald R. Pettit $^{1}$

1. NASA Johnson Space Center, Houston, TX, USA.

2. Voxa, Inc., Seattle, WA, USA.

* Corresponding author: james.e.martinez@nasa.gov

The ISS currently lacks the capability to image and chemically analyze nano-to-micron scale particles from numerous engineering systems. To identify these particles, we must wait for a re-entry vehicle to return them from Low Earth Orbit (LEO) for ground-based SEM/EDS analysis. This may take months, potentially delaying system functionality. Having an EDS equipped SEM (Mochii S) aboard the ISS will accelerate response time thereby enhancing crew and vehicle safety by rapid and accurate identification of microscopic threats, especially in time-critical situations [1].

The Mochii S payload will be located in the Japanese Experiment Module (JEM) powered by 120VAC inverter and connected to ISS Ethernet and Wi-Fi (Fig. 1). To date the Mochii S payload has undergone testing for command and data handling, power quality, flight vibration, and radiation testing at Johnson Space Center (JSC). Mochii's high-RPM rotating vacuum pumps and high voltage systems have been reviewed to meet safety standards by JSC Engineering. System topology in the JEM module has been baselined by ISS Safety and JAXA. Digital controls to and from ISS over Joint Station LAN uplink have been simulated and the latencies and data rates have been found to be sufficient for successful operation of the payload from ground.

Transporting sensitive electron optical instruments aboard a rocket that sustains $7 \mathrm{G}$ of acceleration for 8 minutes and then operating it in the unique micro-g environment is no trivial matter [2]. To meet strict safety requirements and increase robustness for mission success, over 500 unique verifications must be completed before the payload is certified for spaceflight. Two of which will be discussed in detail are: vibroacoustic testing, and magnetic susceptibility shielding and validation.

The term "vibroacoustics" is defined as an environment induced by high-intensity acoustic noise associated with various segments of the flight profile. Vibroacoustics manifests itself throughout the payload in the form of transmitted acoustic excitation and as structure-borne random vibration. Vibration Qualification Tests are performed on prototype hardware that is intended to demonstrate that the test item will function within performance specifications after being exposed to levels which demonstrate margin over the expected flight environment. Durations for qualification testing are defined to demonstrate fatigue-life capability against planned ground testing and exposure to the flight environment. Qualification tests demonstrate, with margin, the design adequacy of the hardware for its intended mission use. Workmanship random vibration and/or acoustic testing are performed to identify latent defects and manufacturing flaws in electrical, electronic, and electromechanical hardware at the component level. The vibroacoustic qualification test durations shall be 2 minutes for the acoustic test and 2 minutes in each of the 3 orthogonal axes for the vibration test (Fig. 2). Acceptance testing is performed to demonstrate that the hardware is acceptable for flight. It also serves as a quality control screen to detect deficiencies in the flight build and is performed at levels and durations which reflect the 
expected flight environment. The vibroacoustic acceptance test durations shall be 1 minute for an acoustic test and 1 minute in each of the 3 orthogonal axes for a vibration test (Fig.3) [3].

During ISS flight, the Mochii S will be exposed to Earth's magnetic field along its orbital path. Each orbital period varies in terms of magnetic field strength and direction (Fig 4). Furthermore, since the Mochii S will be traveling through this varying B-Field at $7.67 \mathrm{~km} / \mathrm{s}$, a resultant Lorentz force will be induced. This force has been determined to be sufficiently high to induce an aberration in the electron beam along its optical path that cannot be corrected with built-in deflectors. The most extreme traversal will occur in the region of the south Atlantic anomaly which has fields dipping as low as $200 \mathrm{mG}$. The fields may exceed $650 \mathrm{mG}$ in the region south of Australia and portions of North America and Siberia (Fig. 5) [4]. Therefore, the Mochii S has been fitted with $\mu$-metal foil to passively shield it from Earth's B-field and reduce beam aberrations or positioning errors. Verification tests of the shielding involve measurements of beam stability in the presence of simulated fields that exceed gradients that the Mochii $\mathrm{S}$ will experience in LEO. A static and dynamic single-axis uniform magnetic field generating apparatus will be constructed using paired Helmholtz coils. The simulated fields will be modeled based on data provided by ESA from the ISS Magnetic Flux Experiment. The test plan will include: baseline, staircase, LEO simulation, accelerated LEO simulation, and hysteresis.

\section{References:}

[1] CS Own et al., Lunar and Planetary Science Conference Proceedings (2018), Contrib. \#2083.

[2] Pettit, D.R. Microsc. Microanal. 21 (Suppl 3), 2015

[3] NASA-STD-7001B, Payload Vibroacoustic Test Criteria

[4] http://eea.spaceflight.esa.int/portal/exp/?id=9446

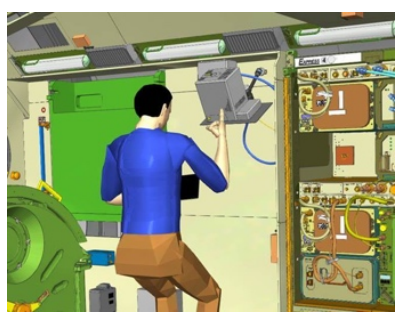

Figure 1. Mochii S topology in the Japanese Experiment Module within ISS.

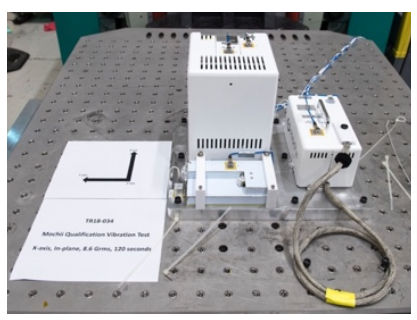

Figure 2. Hard-Mounted Qualification Vibration Test $\mathrm{X}$-Axis.

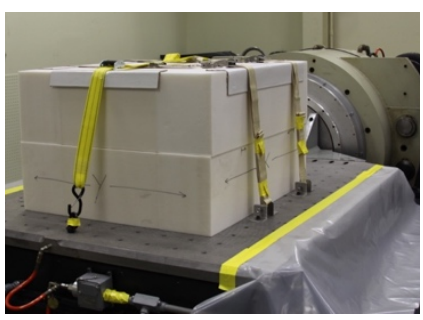

Figure 3. Foam-Mounted Flight Acceptance Vibration Test.

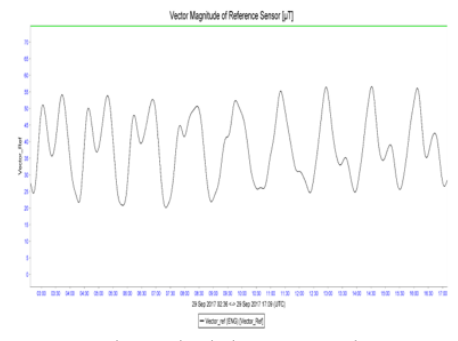

Figure 4. Magnetic Field Intensity measured over 9 Orbital Periods. Fields range from 20 to 55 uT (200 $\mathrm{mG}$ to $550 \mathrm{mG}$ ).

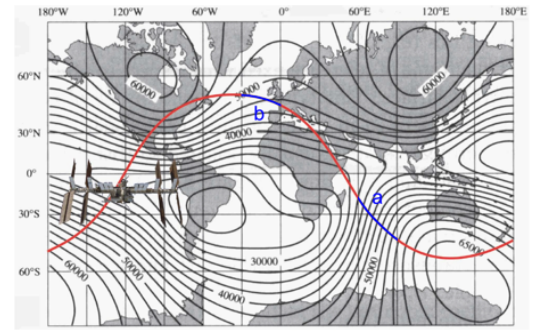

Figure 5. Regions of ISS orbits of interest for testing. a) High gradient region spanning about 7 isopotentials. b) Low gradient region spanning about 1.5 isopotentials. 